\title{
REMARKS ON WEAK SOLUTIONS FOR \\ A NONLOCAL PARABOLIC PROBLEM
}

\section{S. B. DE MENEZES}

Received 7 May 2005; Revised 20 September 2005; Accepted 28 November 2005

Dedicated to Professor L. A. Medeiros on the occasion of his 80th birthday

We prove a result on existence and uniqueness of weak solutions for a diffusion problem associated with nonlinear diffusions of nonlocal type studied by Chipot and Lovat (1999) by an application of the fixed point result of Schauder. Moreover, making use of Faedo-Galerkin approximation, coupled with some technical ideas, we establish a result on existence of periodic solution.

Copyright (c) 2006 Hindawi Publishing Corporation. All rights reserved.

\section{Introduction}

In this work, we are going to study some questions concerning to the existence, uniqueness, and periodic solution for the parabolic problem

$$
\begin{gathered}
u_{t}-a(l(u)) \Delta u+f(u)=h \quad \text { in } Q=\Omega \times(0, T), \\
u(x, t)=0 \quad \text { on } \Sigma=\Gamma \times(0, T), \\
u(x, 0)=u^{0}(x) \quad \text { in } \Omega,
\end{gathered}
$$

where $\Omega$ is a smooth bounded open subset of $\mathbb{R}^{N}$ with regular boundary $\Gamma$. In problem (1.1) $a$ and $f$ are both continuous functions, whose properties will be introduced when necessary, $l: L^{2}(\Omega) \rightarrow \mathbb{R}$ is a nonlinear form, $h \in L^{2}\left(0, T ; H^{-1}(\Omega)\right)$, and $T>0$ is some fixed time. System (1.1) is studied, for instance, in papers of Chipot and Lovat [4] in case $f=f(x)$ depends only on the variable $x$. In this work, we are going to present a simple extension of the results contained in [4], in which $f=f(u)$ depends on the state $u$, where we study, among other things, the case in which $u$ is periodic. This kind of problems, besides its mathematical motivation because of presence of the nonlocal term $a=a(l(u))$, arises from physical situations related to migration of a population of bacterias in a container in which the velocity of migration $\vec{v}=a \nabla u$ depends on the global population in a subdomain $\Omega^{\prime} \subset \Omega$ given by $a=a\left(\int_{\Omega^{\prime}} u d x\right)$. For more details see [4] and the references cited in this paper. Many books have dealt with parabolic equations and asymptotic analysis. See, for example, Amann [1], Haraux [5], Pao [6], and Zeidler [7] and 
the references therein. However, because of the nonlocal term $a$ we cannot, in the present problem, make a direct adaptation of the classical techniques. In view of this we have to appeal to another device in order to obtain results similar to those in which appear only local terms. In particular, our approach rests heavily on the ideas developed by Chipot and Lovat [4]. This work is organized as follows. In Section 2, we prove basic results on existence and uniqueness of weak solution for problem (1.1). The methodology of the proof of our results is based on the fixed point argument. Finally, in Section 3, we will prove the existence and uniqueness of periodic weak solution for problem (1.1). We use Faedo-Galerkin method and Brower fixed point theorem plus also some technical ideas.

\section{Results on existence and uniqueness}

The first object of this work is to prove existence and uniqueness for the problem (1.1). In fact, concerning problem (1.1), we will suppose that $a: \mathbb{R} \rightarrow \mathbb{R}$ is continuous and that for some constants $m, M$,

$$
\begin{gathered}
0<m \leq a(\xi) \leq M, \quad \forall \xi \in \mathbb{R}, \\
l: L^{2}(\Omega) \longrightarrow \mathbb{R} \text { is a continuous nonlinear form, }
\end{gathered}
$$

that is, there is $g \in L^{2}(\Omega)$ such that $l(u)=l_{g}(u)=\int_{\Omega} g(x) u(x) d x$ for all $u \in L^{2}(\Omega)$ and $f: \mathbb{R} \rightarrow \mathbb{R}$ is a Lipschitz continuous function, that is, there exists $\gamma>0$ such that

$$
|f(s)-f(t)| \leq \gamma|s-t|, \quad \forall s, t \in \mathbb{R} .
$$

Moreover assume that $f(0)=0, f^{\prime}(0)$ exists.

In this section, we present some notation that will be used throughout this work. By $\langle\cdot, \cdot\rangle$ we will represent the duality pairing between $X$ and $X^{\prime}, X^{\prime}$ being the topological dual of the space $X$, and by $C$ (sometimes $C_{1}, C_{2}, \ldots$ ) we denote various positive constants. We represent by $H^{m}(\Omega)$ the usual Sobolev space of order $m$, by $H_{0}^{m}(\Omega)$ the closure of $C_{0}^{\infty}(\Omega)$ in $H^{m}(\Omega)$, and by $L^{2}(\Omega)$ the class of square Lebesgue integrable real functions. In particular, $H_{0}^{1}(\Omega)$ has inner product $((\cdot, \cdot))$ and norm $\|\cdot\|$ given by $((u, v))=\int_{\Omega} \nabla u$. $\nabla v d x ;\|u\|^{2}=\int_{\Omega}|\nabla u|^{2} d x$. For the Hilbert space $L^{2}(\Omega)$ we represent its inner and norm, respectively, by $(\cdot, \cdot)$ and $|\cdot|$, defined by $(u, v)=\int_{\Omega} u v d x ;|u|^{2}=\int_{\Omega}|u|^{2} d x$. We have our first result.

THEOREM 2.1. If (2.1)-(2.3) hold, then for

$$
u^{0} \in L^{2}(\Omega), \quad h \in L^{2}\left(0, T, H^{-1}(\Omega)\right)
$$

there exists a function $u$ such that

$$
\begin{gathered}
u \in L^{2}\left(0, T, H_{0}^{1}(\Omega)\right) \cap C\left([0, T], L^{2}(\Omega)\right), \quad u_{t} \in L^{2}\left(0, T, H^{-1}(\Omega)\right), \\
u(x, 0)=u^{0}, \\
\frac{d}{d t}(u, v)+a(l(u)) \int_{\Omega} \nabla u \cdot \nabla v d x+\int_{\Omega} f(u) v d x=\langle h, v\rangle,
\end{gathered}
$$

for all $v \in H_{0}^{1}(\Omega)$, where (2.7) must be understood as an equality in $\mathscr{D}^{\prime}(0, T)$. 
Proof. We argue using the Schauder fixed point theorem. For that purpose, consider $w \in$ $L^{2}\left(0, T ; L^{2}(\Omega)\right)$ and $u=N(w)$ the unique solution to "linearized" problem

$$
\begin{gathered}
u \in L^{2}\left(0, T, H_{0}^{1}(\Omega)\right) \cap C\left([0, T], L^{2}(\Omega)\right), \quad u_{t} \in L^{2}\left(0, T, H^{-1}(\Omega)\right), \\
u(x, 0)=u^{0}, \\
\frac{d}{d t}(u, v)+a(l(w)) \int_{\Omega} \nabla u \cdot \nabla v d x+\int_{\Omega} b(x, t, w) u v d x=\langle h, v\rangle,
\end{gathered}
$$

in $\mathscr{D}^{\prime}(0, T)$ for all $v \in H_{0}^{1}(\Omega)$.

Here $b: \Omega \times \mathbb{R}^{+} \times \mathbb{R} \rightarrow \mathbb{R}$ is defined by

$$
b(x, t, \eta)=\left\{\begin{array}{cc}
\frac{f(\eta)}{\eta} & \text { for } \eta \neq 0 \\
f^{\prime}(0) & \text { for } \eta=0
\end{array}\right.
$$

We note that the mapping

$$
t \longmapsto l(w)
$$

is measurable, and due to (2.1)-(2.3), so is

$$
t \longmapsto a(l(w)) .
$$

We also note that $a(l(w)) \in L^{\infty}(0, T)$, and $b(x, t, \eta)$ is continuous, a.e. $(x, t) \in \Omega \times \mathbb{R}^{+}$, and measurable for all $\eta \in \mathbb{R}$ and

$$
|b(x, t, \eta)| \leq C \quad \text { a.e. }(x, t) \in \Omega \times \mathbb{R}^{+}, \forall \eta \in \mathbb{R} .
$$

We know that such a $u=N(w)$ exists (see, e.g., [3]). Thus we would like to show that the mapping

$$
w \longmapsto N(w)
$$

from $L^{2}\left(0, T ; L^{2}(\Omega)\right)$ into itself has a fixed point - this will be clearly a solution to our problem.

First let us remark that

$$
u_{t}-a(l(w)) \triangle u+b(x, t, w) u=h \quad \text { in } L^{2}\left(0, T ; H^{-1}(\Omega)\right),
$$

and thus for every $v \in L^{2}\left(0, T ; H_{0}^{1}(\Omega)\right)$,

$$
\left\langle u_{t}, v\right\rangle+a(l(w))(\nabla u, \nabla v)+b(x, t, w)(u, v)=\langle h, v\rangle \quad \text { a.e. } t \in(0, T) .
$$

Taking $v=u$ in (2.17), and in view of (2.1) and (2.14), one gets

$$
\frac{1}{2} \frac{d}{d t}|u(t)|^{2}+\left.m|| u(t)\right|^{2} \leq C|u(t)|^{2}+\| u(t)|||h|_{H^{-1}(\Omega)} .
$$


4 Remarks on weak solutions for a nonlocal parabolic problem

Applying Young's inequality, we obtain

$$
\frac{1}{2} \frac{d}{d t}|u(t)|^{2}+m\|u(t)\|^{2} \leq C|u(t)|^{2}+\frac{m}{2}\|u(t)\|^{2}+\frac{1}{2 m}|h|_{H^{-1}(\Omega)}^{2},
$$

that is,

$$
\frac{1}{2} \frac{d}{d t}|u(t)|^{2}+\frac{m}{2} \|\left. u(t)\right|^{2} \leq C|u(t)|^{2}+\frac{1}{2 m}|h|_{H^{-1}(\Omega)}^{2} .
$$

Integrating it over $(0, t)$ and employing Gronwall's lemma, we obtain

$$
|u|_{L^{2}\left(0, T ; H_{0}^{1}(\Omega)\right)} \leq C, \quad|u|_{L^{2}\left(0, T ; L^{2}(\Omega)\right)} \leq C .
$$

Setting

$$
B=\left\{\left.v \in L^{2}\left(0, T ; L^{2}(\Omega)\right)|| v\right|_{L^{2}\left(0, T ; L^{2}(\Omega)\right)} \leq C\right\},
$$

it follows that $w \mapsto u=N(w)$ is a mapping from $B$ into itself. The arguments above show that when $w$ lies in a bounded set $B$ of $L^{2}\left(0, T ; L^{2}(\Omega)\right), u=N(w)$ also lies in a bounded set of $L^{2}\left(0, T ; L^{2}(\Omega)\right)$. We have to show that $N(B)$ is relatively compact in $L^{2}\left(0, T ; L^{2}(\Omega)\right)$. Indeed, going back to (2.10), we have

$$
\left|u_{t}\right|_{L^{2}\left(0, T ; H^{-1}(\Omega)\right)} \leq C
$$

Then $u$ belongs to $W\left(0, T, H_{0}^{1}(\Omega), H^{-1}(\Omega)\right)$. We recall that

$$
W(0, T, X, Y)=\left\{v \in L^{2}(0, T ; X) \mid v_{t} \in L^{2}(0, T ; Y)\right\}
$$

Thus, the compactness of $N$ is a consequence of the compactness of the embedding of $W\left(0, T, H_{0}^{1}(\Omega), H^{-1}(\Omega)\right)$ into $L^{2}\left(0, T ; L^{2}(\Omega)\right)$ (Aubin-Lions compactness result). In order to be able to apply the Schauder fixed point theorem, we now just need to prove that $N$ is continuous from $B$ into itself. For that let $\left(w_{n}\right)$ be a sequence in $B$ such that

$$
w_{n} \longrightarrow w \quad \text { in } L^{2}\left(0, T ; L^{2}(\Omega)\right) .
$$

Set $u_{n}=N\left(w_{n}\right)$. From (2.25) we derive that

$$
l\left(w_{n}\right) \longrightarrow l(w) \quad \text { in } L^{2}(0, T)
$$

By the estimates above and Aubin-Lions compactness result we can find $u_{\infty} \in W(0, T$, $\left.H_{0}^{1}(\Omega), H^{-1}(\Omega)\right)$ and a subsequence from $n$ that we will label also $n$ such that

$$
\begin{gathered}
u_{n} \longrightarrow u_{\infty} \quad \text { weakly in } W\left(0, T, H_{0}^{1}(\Omega), H^{-1}(\Omega)\right), \\
u_{n} \longrightarrow u_{\infty} \quad \text { strongly in } L^{2}\left(0, T ; L^{2}(\Omega)\right), \\
l\left(w_{n}\right) \longrightarrow l(w) \quad \text { a.e. } t \in(0, T) .
\end{gathered}
$$


By continuity of $a$ we obtain

$$
a\left(l\left(w_{n}\right)\right) \longrightarrow a(l(w)) \text { a.e. } t \in(0, T) .
$$

From (2.10) we have that

$$
\begin{gathered}
-\int_{0}^{T} \int_{\Omega} u_{n} v \varphi^{\prime}(t) d x d t+\int_{0}^{T} \int_{\Omega} a\left(l\left(w_{n}\right)\right) \nabla u_{n} \cdot \nabla v \varphi(t) d x d t \\
+\int_{0}^{T} \int_{\Omega} b\left(x, t, w_{n}\right) u_{n} v \varphi(t) d x d t=\int_{0}^{T}\langle h, v\rangle \varphi(t) d t
\end{gathered}
$$

for any $\varphi \in \mathscr{D}(0, T), v \in H_{0}^{1}(\Omega)$.

By the Lebesgue's dominated convergence theorem we have

$$
\begin{gathered}
\varphi(t) b\left(x, t, w_{n}\right) v \longrightarrow \varphi(t) b(x, t, w) v, \\
\varphi(t) a\left(l\left(w_{n}\right)\right) \frac{\partial v}{\partial x_{i}} \longrightarrow \varphi(t) a(l(w)) \frac{\partial v}{\partial x_{i}},
\end{gathered}
$$

in $L^{2}\left(0, T ; L^{2}(\Omega)\right)$. Taking limit in both sides of (2.31), we obtain that $u_{\infty}$ satisfies $(2.8)$ and (2.10). By (2.28) one can assume without loss of generality that

$$
u_{n}(t) \longrightarrow u_{\infty}(t) \quad \text { in } L^{2}(\Omega) \text {, a.e. } t \in(0, T) \text {. }
$$

Thus

$$
u_{\infty}(0)=u^{0}
$$

Then by the uniqueness of the solution to (2.8)-(2.10) we have that $u_{\infty}=u$. Since any subsequence of $u_{n}$ has the same limit,

$$
u_{n}=N\left(w_{n}\right) \longrightarrow u=N(w) \quad \text { in } L^{2}\left(0, T ; L^{2}(\Omega)\right),
$$

which concludes the proof of Theorem 2.1.

Next, we will prove the following result.

Theorem 2.2. Assume that a is Lipschitz continuous in the sense that there exists a constant A such that

$$
\left|a(t)-a\left(t^{\prime}\right)\right| \leq A\left|t-t^{\prime}\right|, \quad \forall t, t^{\prime} \in \mathbb{R} .
$$

Then under the assumptions of Theorem 2.1, the problem (1.1) has a unique solution.

Proof. Let us denote by $u_{1}$ and $u_{2}$ two solutions of (1.1). Thus

$$
\begin{gathered}
\left(\frac{d}{d t}\left(u_{1}-u_{2}\right), v\right)+a\left(l\left(u_{1}\right)\right) \int_{\Omega} \nabla u_{1} \cdot \nabla v d x-a\left(l\left(u_{2}\right)\right) \\
\times \int_{\Omega} \nabla u_{2} \cdot \nabla v d x+\int_{\Omega}\left(f\left(u_{1}\right)-f\left(u_{2}\right)\right) v d x=0 .
\end{gathered}
$$


6 Remarks on weak solutions for a nonlocal parabolic problem

From (2.3) we obtain

$$
\begin{aligned}
& \left(\frac{d}{d t}\left(u_{1}-u_{2}\right), v\right)+a\left(l\left(u_{1}\right)\right) \int_{\Omega} \nabla\left(u_{1}-u_{2}\right) \cdot \nabla v d x \\
& \quad \leq\left(a\left(l\left(u_{2}\right)\right)-a\left(l\left(u_{1}\right)\right)\right) \int_{\Omega} \nabla u_{2} \cdot \nabla v d x+\gamma \int_{\Omega}\left|u_{1}-u_{2}\right| v d x
\end{aligned}
$$

Taking $v=\left(u_{1}-u_{2}\right)(t)$, for a.e. $t$, one gets

$$
\begin{aligned}
& \frac{1}{2} \frac{d}{d t}\left|u_{1}-u_{2}\right|^{2}+a\left(l\left(u_{1}\right)\right)|| u_{1}-\left.u_{2}\right|^{2} \\
& \quad \leq\left|a\left(l\left(u_{2}\right)\right)-a\left(l\left(u_{1}\right)\right)\right| \int_{\Omega}\left|\nabla u_{2}\right|\left|\nabla\left(u_{1}-u_{2}\right)\right| d x+\gamma\left|u_{1}-u_{2}\right|^{2}
\end{aligned}
$$

So by the Cauchy-Schwarz inequality and by using (2.1)-(2.2) and (2.36), one gets

$$
\begin{aligned}
\frac{1}{2} \frac{d}{d t} \mid & u_{1}-\left.u_{2}\right|^{2}+m|| u_{1}-u_{2} \|^{2} \\
& \leq A\left|l\left(u_{2}\right)-l\left(u_{1}\right)\right||| u_{2}|||| u_{1}-u_{2}||+\gamma\left|u_{1}-u_{2}\right|^{2} \\
& \leq C\left|u_{1}-u_{2}\right||| u_{2}|||| u_{1}-u_{2}||+\gamma\left|u_{1}-u_{2}\right|^{2} .
\end{aligned}
$$

Then, applying Young's inequality, we obtain

$$
\begin{aligned}
\frac{1}{2} \frac{d}{d t} & \left|u_{1}-u_{2}\right|^{2}+m|| u_{1}-u_{2} \|^{2} \\
& \leq \frac{m}{2}\left\|u_{1}-u_{2}\right\|^{2}+\frac{1}{2 m} C^{2}|| u_{2} \|^{2}\left|u_{1}-u_{2}\right|^{2}+\gamma\left|u_{1}-u_{2}\right|^{2} \\
& =\frac{m}{2}\left\|u_{1}-u_{2}\right\|^{2}+\left(\frac{C^{2}}{2 m}\left\|u_{2}\right\|^{2}+\gamma\right)\left|u_{1}-u_{2}\right|^{2}
\end{aligned}
$$

which gives

$$
\frac{d}{d t}\left|u_{1}-u_{2}\right| \leq \zeta(t)\left|u_{1}-u_{2}\right|^{2}
$$

where the function $\zeta(t)$ belongs to $L^{1}(0, T)$. So this reads after multiplication by

$$
\begin{gathered}
\exp \left(-\int_{0}^{t} \zeta(s) d s\right) \\
\frac{d}{d t}\left(\exp \left(-\int_{0}^{t} \zeta(s) d s\right)\left|u_{1}-u_{2}\right|^{2}\right) \leq 0 .
\end{gathered}
$$

Since this last function is nonincreasing and vanishes at $0\left(u_{1}(0)=u_{2}(0)\right)$, the result follows. 


\section{Periodic solution}

The second object of this work is to show the existence of periodic solution of problem (1.1). For this purpose, in addition to (2.1)-(2.3), we assume that

$$
m \lambda_{1}-2 \gamma>0
$$

with $\lambda_{1}$ the first eigenvalue of $\left(-\triangle, H_{0}^{1}(\Omega)\right)$. We make use of Faedo-Galerkin approximation and Brower fixed point theorem. The result on the periodic solution is given by the following theorem.

Theorem 3.1. Under the assumptions of Theorem 2.2, assume that (3.1) holds. If $h \in L^{2}$ $\left(0, T, H^{-1}(\Omega)\right)$, then there exists a unique solution $u$ of the periodic problem

$$
\begin{gathered}
u \in L^{2}\left(0, T, H_{0}^{1}(\Omega)\right) \cap C\left([0, T], L^{2}(\Omega)\right), \quad u_{t} \in L^{2}\left(0, T, H^{-1}(\Omega)\right), \\
\frac{d}{d t}(u, v)+a(l(u)) \int_{\Omega} \nabla u \cdot \nabla v d x+\int_{\Omega} f(u) v d x=\langle h, v\rangle,
\end{gathered}
$$

for all $v \in H_{0}^{1}(\Omega)$, in the sense of $\mathscr{D}^{\prime}(0, T)$,

$$
u(0)=u(T) \quad \text { in } L^{2}(\Omega)
$$

Proof. We employ the Faedo-Galerkin method. Let $\left(\omega_{j}\right)_{j \in \mathbb{N}}$ be a Hilbertian basis of $H_{0}^{1}(\Omega)$ (cf. Brezis [2]). Represent by $\mathbb{V}_{j}$ the subspace of $H_{0}^{1}(\Omega)$ generated by $\left\{\omega_{1}, \ldots, \omega_{j}\right\}$ and let us consider the approximate problem given by

$$
\begin{gathered}
u_{j} \in \mathbb{V}_{j}, \\
\left(u_{j}^{\prime}, v\right)+a\left(l\left(u_{j}\right)\right)\left(\nabla u_{j}, \nabla v\right)+\left(f\left(u_{j}\right), v\right)=\langle h, v\rangle, \quad \forall v \in \mathbb{V}_{j}, \\
u_{j}(0)=u_{j}^{0} \longrightarrow u^{0} \quad \text { strongly in } L^{2}(\Omega) .
\end{gathered}
$$

The system of ordinary differential equations (3.4)-(3.6) has a local solution on an interval $\left[0, t_{m}\left[, 0<t_{m}<T\right.\right.$. We now have to establish an estimate that permits to extend the solution to the whole interval $[0, T]$. Taking $v=u_{j}$ in (3.5) and in view of (2.1)-(2.3), one gets

$$
\frac{1}{2} \frac{d}{d t}\left|u_{j}(t)\right|^{2}+m|| u_{j}(t)\left\|^{2} \leq \gamma\left|u_{j}(t)\right|^{2}+\right\| u_{j}|||h|_{H^{-1}(\Omega)} .
$$

Thanks to Young's inequality, one gets

$$
\frac{1}{2} \frac{d}{d t}\left|u_{j}(t)\right|^{2}+\frac{m}{2} \|\left. u_{j}(t)\right|^{2} \leq \gamma\left|u_{j}(t)\right|^{2}+\frac{1}{2 m}|h|_{H^{-1}(\Omega)}^{2} .
$$


8 Remarks on weak solutions for a nonlocal parabolic problem

As in the proof of Theorem 2.1, integrating it over $(0, t)$ and employing Gronwall's lemma, we obtain

$$
\begin{gathered}
\left|u_{j}\right|_{L^{2}\left(0, T ; H_{0}^{1}(\Omega)\right)} \leq C, \\
\left|u_{j}\right|_{L^{\infty}\left(0, T ; L^{2}(\Omega)\right)} \leq C, \\
\left|\partial_{t} u_{j}\right|_{L^{2}\left(0, T ; H^{-1}(\Omega)\right)} \leq C .
\end{gathered}
$$

In view of the estimates above, we can extend the approximate solution $u_{j}(t)$ to $[0, T]$.

Next, let us consider

$$
u_{j}(0)=u_{j}^{0} \in B_{0}(R) \cap \mathbb{V}_{j},
$$

where $B_{0}(R)=\left\{u \in L^{2}(\Omega) ;|u|_{L^{2}(\Omega)}<R\right\}$, with $R$ a positive constant.

Let $\lambda_{1}$ be the first eigenvalue of $\left(-\triangle, H_{0}^{1}(\Omega)\right)$. Then, thanks to Poincare's inequality and (3.8), we obtain

$$
\frac{d}{d t}\left|u_{j}(t)\right|^{2}+\left(m \lambda_{1}-2 \gamma\right)\left|u_{j}(t)\right|^{2} \leq \frac{1}{2 m}|h|_{H^{-1}(\Omega)}^{2}
$$

We note that, by hypothesis (3.1), $m \lambda_{1}-2 \gamma>0$ and thus

$$
\frac{d}{d t}\left|u_{j}(t)\right|^{2}+C\left|u_{j}(t)\right|^{2} \leq C|h|_{H^{-1}(\Omega)}^{2}
$$

We multiply both sides of (3.14) by $e^{C t}$ and we integrate on $[0, t)$ to obtain

$$
\left|u_{j}(t)\right|^{2} \leq e^{-C t}\left|u_{j}(0)\right|^{2}+C \int_{0}^{T} e^{-C(s-T)}|h(s)|_{H^{-1}(\Omega)}^{2} d s .
$$

Since $h \in L^{2}\left(0, T, H^{-1}(\Omega)\right)$, one has

$$
\left|u_{j}(t)\right|^{2} \leq \theta(t)\left|u_{j}(0)\right|^{2}+C,
$$

for all $0 \leq t \leq T$, with $\theta(t)=e^{-C t}$ and $C=\int_{0}^{T} e^{-C(s-T)}|h(s)|_{H^{-1}(\Omega)}^{2} d s$. We note that $0<$ $\theta(t)<1$. Then,

$$
\left|u_{j}(T)\right|^{2} \leq \theta\left|u_{j}(0)\right|^{2}+C
$$

with $\theta=\theta(T)$ constant, $0<\theta<1$. Choosing $R>0$ such that $C /(1-\theta)<R^{2}$, we have that

$$
\left|u_{j}(T)\right|^{2} \leq R^{2}
$$

Thus, for each $u_{j}^{0} \in B_{0}(R) \cap \mathbb{V}_{j}$ there exists a solution $u_{j}(t)$ of the approximating problem (3.4)-(3.6) and, furthermore, $u_{j}(t)$ satisfies (3.18). So, we have defined a mapping

$$
\begin{gathered}
\tau: B_{0}(R) \cap \mathbb{V}_{j} \longrightarrow B_{0}(R) \cap \mathbb{V}_{j}, \\
u_{j}^{0} \longmapsto \tau\left(u_{j}^{0}\right)=u_{j}(T) .
\end{gathered}
$$

We are ready to establish the following lemma. 
Lemma 3.2. Assume that hypotheses of Theorem 2.2 hold. Then the mapping $\tau$ is continuous. Proof. Let us consider $u_{1_{j}}^{0}, u_{2_{j}}^{0}$ in $B_{0}(R) \cap \mathbb{V}_{j}$ and $u_{1}, u_{2}$ the corresponding solutions of (3.4)-(3.6). From (2.41) we obtain

$$
\frac{1}{2} \frac{d}{d t}\left|u_{1_{j}}-u_{2_{j}}\right|^{2}+\frac{m}{2}|| u_{1_{j}}-\left.u_{2_{j}}\right|^{2} \leq C\left|u_{1_{j}}-u_{2_{j}}\right|^{2}
$$

since, by Theorem 2.1, $\left\|u_{2 j}\right\|<C$. By Gronwall's lemma, one gets

$$
\left|u_{1_{j}}(T)-u_{2_{j}}(T)\right| \leq C\left|u_{1_{j}}^{0}-u_{2_{j}}^{0}\right| \text {. }
$$

Returning to (3.20) and using the equivalence between the norms in $\mathbb{V}_{j}$, we obtain

$$
\left|u_{1_{j}}(T)-u_{2_{j}}(T)\right|_{\mathbb{v}_{j}} \leq C\left|u_{1_{j}}^{0}-u_{2_{j}}^{0}\right|_{\mathbb{V}_{j}}
$$

and, therefore, Lemma 3.2 follows. Returning to the map $\tau$ and using Lemma 3.2, we obtain, from the fixed Brower theorem, that there exists $u_{1_{j}}^{0} \in \mathbb{V}_{j}$ such that

$$
\tau\left(u_{1_{j}}^{0}\right)=u_{1_{j}}^{0} .
$$

Thus,

$$
u_{1_{j}}(0)=u_{1_{j}}(T)
$$

where $u_{1_{j}}$ is the solution of the approximate problem (3.4)-(3.6) with initial datum $u_{1_{j}}^{0}$. Since the estimates (3.9)-(3.11) were uniform in $j$, we can see that exist a subsequence of $\left(u_{1_{j}}\right)$, again called $\left(u_{1_{j}}\right)$, and a function $u$ such that

$$
\begin{gathered}
u_{1_{j}} \stackrel{*}{\longrightarrow} u \quad \text { weak star in } L^{\infty}\left(0, T, L^{2}(\Omega)\right), \\
u_{1_{j}} \longrightarrow u \quad \text { weakly in } L^{2}\left(0, T, H_{0}^{1}(\Omega)\right), \\
\partial_{t} u_{1_{j}} \longrightarrow \partial_{t} u \quad \text { weakly in } L^{2}\left(0, T, H^{-1}(\Omega)\right), \\
\frac{d}{d t}(u, v)+a(l(u)) \int_{\Omega} \nabla u \cdot \nabla v d x+\int_{\Omega} f(u) v d x=\langle h, v\rangle,
\end{gathered}
$$

for all $v \in H_{0}^{1}(\Omega)$, in the sense of $\mathscr{D}^{\prime}(0, T)$.

Finally, we will now prove that

$$
u(0)=u(T) .
$$

Indeed, by (3.26) we have

$$
\int_{0}^{T}\left(u_{1_{j}}(t), v\right) \psi^{\prime}(t) d t \longrightarrow \int_{0}^{T}(u(t), v) \psi^{\prime}(t) d t,
$$

for all $v \in H_{0}^{1}(\Omega)$ and $\psi \in H_{0}^{1}(0, T)$, with $\psi(T)=0$. 
10 Remarks on weak solutions for a nonlocal parabolic problem

Also by (3.27) we obtain

$$
\int_{0}^{T} \frac{d}{d t}\left(u_{1_{j}}(t), v\right) \psi(t) d t \rightarrow \int_{0}^{T} \frac{d}{d t}(u(t), v) \psi(t) d t
$$

for all $v \in H_{0}^{1}(\Omega)$ and $\psi \in H_{0}^{1}(0, T)$, with $\psi(T)=0$.

It follows from (3.30) and (3.31) that

$$
\int_{0}^{T} \frac{d}{d t}\left[\left(u_{1_{j}}(t), v\right) \psi(t)\right] d t \longrightarrow \int_{0}^{T} \frac{d}{d t}[(u(t), v) \psi(t)] d t,
$$

that is,

$$
\left(u_{1_{j}}(0), v\right) \longrightarrow(u(0), v), \quad \forall v \in H_{0}^{1}(\Omega)
$$

We use the preceding argument with $\psi \in H_{0}^{1}(\Omega), \psi(0)=0$, to get

$$
\left(u_{1_{j}}(T), v\right) \longrightarrow(u(T), v), \quad \forall v \in H_{0}^{1}(\Omega) .
$$

Since $u_{1_{j}}(0)=u_{1_{j}}(T)$, it follows from (3.33) and (3.34) that $u(T)=u(0)$ in $L^{2}(\Omega)$, which concludes the proof of our Theorem 3.1.

\section{References}

[1] H. Amann, Linear and Quasilinear Parabolic Problems. Vol. I, Monographs in Mathematics, vol. 89, Birkhäuser Boston, Massachusetts, 1995.

[2] H. Brezis, Analyse Fonctionnelle: Théorie et Applications [Functional Analysis: Theory and Applications], Masson, Paris, 1983.

[3] M. Chipot, Elements of Nonlinear Analysis, Birkhäuser Advanced Texts, Birkhäuser, Basel, 2000.

[4] M. Chipot and B. Lovat, On the asymptotic behaviour of some nonlocal problems, Positivity 3 (1999), no. 1, 65-81.

[5] A. Haraux, Systèmes Dynamiques Dissipatifs et Applications [Dissipative Dynamical Systems and Applications], Research in Applied Mathematics, vol. 17, Masson, Paris, 1991.

[6] C. V. Pao, Nonlinear Parabolic and Elliptic Equations, Plenum Press, New York, 1992.

[7] E. Zeidler, Nonlinear Functional Analysis and Its Applications. II/B. Nonlinear Monotone Operator, Springer, New York, 1990.

S. B. de Menezes: Departamento de Matemática, Universidade Federal do Pará, 66075-110 Belém, Pará, Brazil

E-mail address: silvano@ufpa.br 


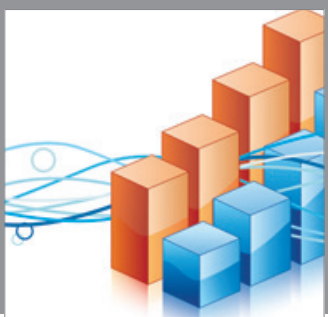

Advances in

Operations Research

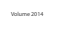

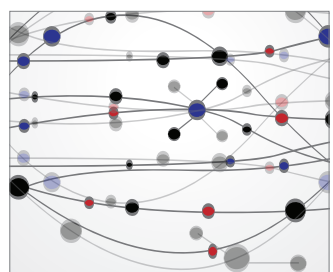

\section{The Scientific} World Journal
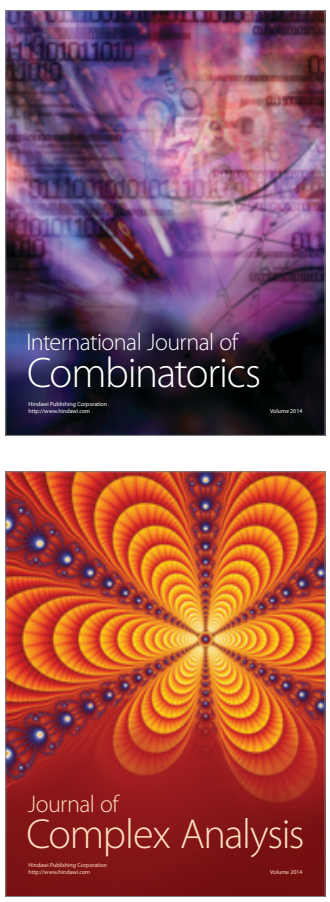

International Journal of

Mathematics and

Mathematical

Sciences
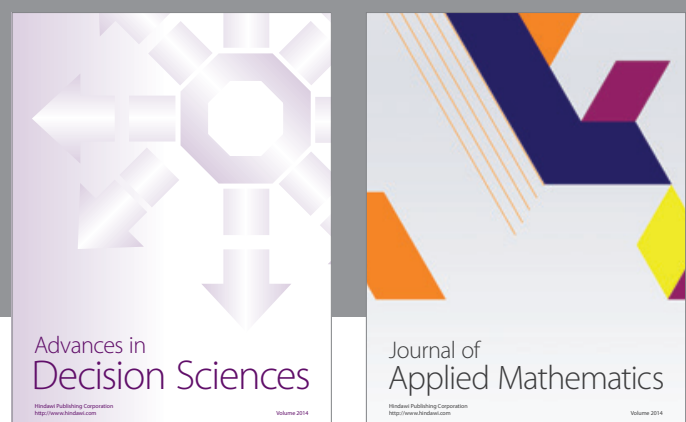

Journal of

Applied Mathematics
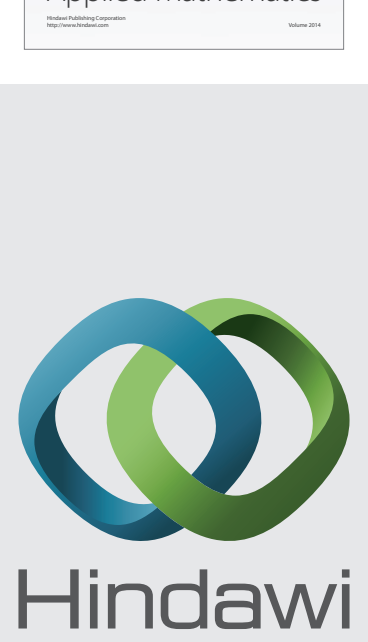

Submit your manuscripts at http://www.hindawi.com
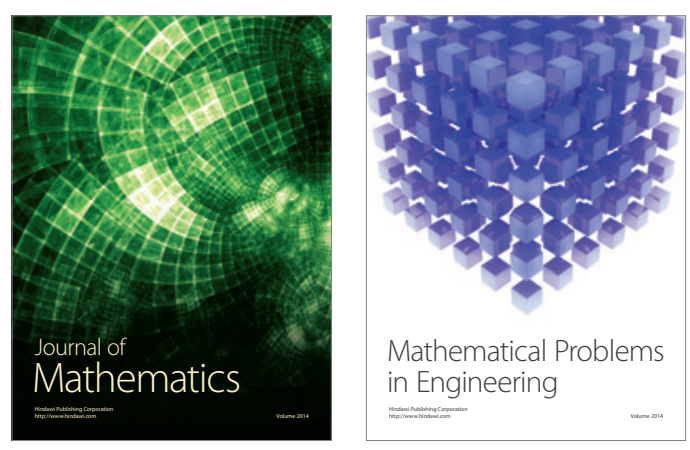

Mathematical Problems in Engineering
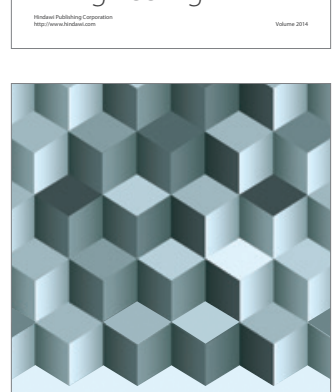

Journal of

Function Spaces
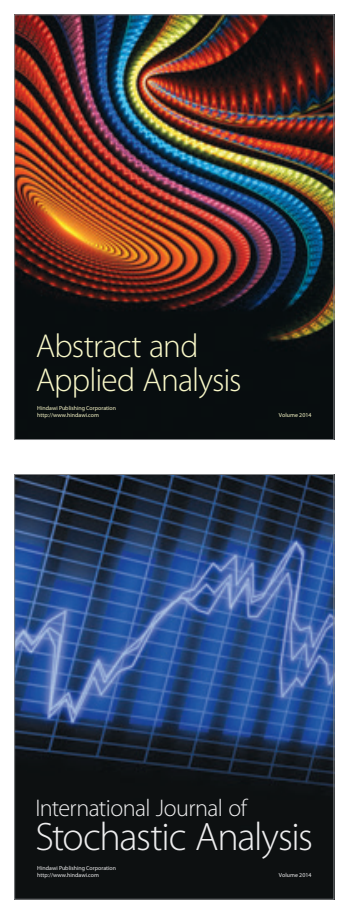

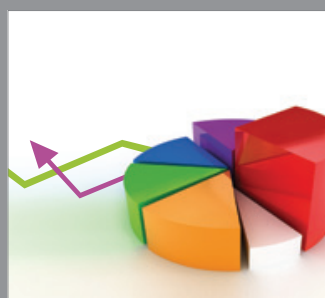

ournal of

Probability and Statistics

Promensencen
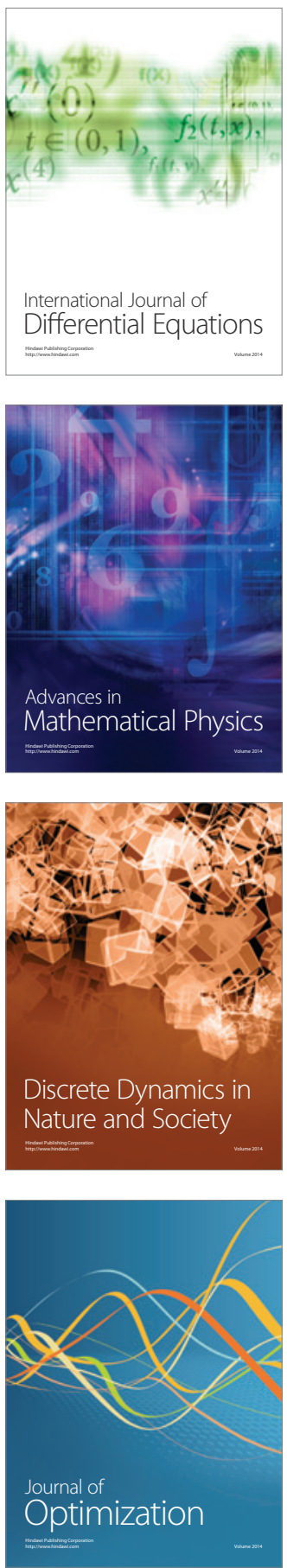